PROCEEDINGS OF THE

AMERICAN MATHEMATICAL SOCIETY

Volume 135, Number 9, September 2007, Pages 2723-2731

S 0002-9939(07)08880-6

Article electronically published on February 16, 2007

\title{
ON POINTED HOPF ALGEBRAS ASSOCIATED TO SOME CONJUGACY CLASSES IN $\mathbb{S}_{n}$
}

\author{
NICOLÁS ANDRUSKIEWITSCH AND SHOUCHUAN ZHANG
}

(Communicated by Martin Lorenz)

\begin{abstract}
We show that any pointed Hopf algebra with infinitesimal braiding associated to the conjugacy class of $\pi \in \mathbb{S}_{n}$ is infinite-dimensional, if either the order of $\pi$ is odd, or all cycles in the decomposition of $\pi$ as a product of disjoint cycles have odd order except for exactly two transpositions.
\end{abstract}

\section{INTRODUCTION}

The purpose of this article is to contribute to the classification of finite-dimensional complex pointed Hopf algebras $H$ with $G(H)=\mathbb{S}_{n}$. Although substantial progress has been made in the classification of finite-dimensional complex pointed Hopf algebras with abelian group [AS4, not much is known about the non-abelian case. Our approach fits into the framework of the Lifting Method [AS1, AS3]; we shall freely use the notation and results from loc. cit. Given a finite group $G$, the key step in the classification of finite-dimensional complex pointed Hopf algebras $H$ with $G(H)=G$ is the determination of all Yetter-Drinfeld modules $V$ over the group algebra of $G$ such that the Nichols algebra is finite-dimensional. If $G$ is abelian, this reduces to the study of Nichols algebras of diagonal type; general results were reached in this situation in $\mathrm{AS2}, \mathrm{H}$ for braided vector spaces of Cartan type. If $G$ is not abelian, then very few examples have been computed explicitly in the literature; see [FK], MS, G1, AG2]. Our viewpoint in the present paper is to deduce that some Nichols algebras over non-abelian groups are infinite-dimensional from those results on Nichols algebras of diagonal type. This idea appeared first in [G1. More precisely, recall that an irreducible Yetter-Drinfeld module over the group algebra of $G$ is determined by a conjugacy class $\mathcal{C}$ of $G$ and an irreducible representation $\rho$ of the centralizer $G^{s}$ of a fixed $s \in \mathcal{C}$. We seek conditions on $\mathcal{C}$ and $\rho$ implying that the dimension of the Nichols algebra $\mathfrak{B}(\mathcal{C}, \rho)$ is infinite. We concentrate on the central example $G=\mathbb{S}_{n}$. Up to now, the only pairs $(\mathcal{C}, \rho)$ for $\mathbb{S}_{n}$ such that the dimension $\mathfrak{B}(\mathcal{C}, \rho)$ is known are as follows.

- If either $\mathcal{C}$ or $\rho$ is trivial, then the dimension of $\mathfrak{B}(\mathcal{C}, \rho)$ is infinite (this is easy and holds for any $G$ ).

Received by the editors November 1, 2005 and, in revised form, May 25, 2006.

2000 Mathematics Subject Classification. Primary 16W30.

The work of the first author was partially supported by CONICET, Fund. Antorchas, Agencia Córdoba Ciencia, TWAS (Trieste), ANPCyT and Secyt (UNC). Results of this paper were obtained during a visit of the first author to the Hunan University, Changsha (China).

(C)2007 American Mathematical Society Reverts to public domain 28 years from publication 2723 
- If $\mathcal{C}=\mathcal{O}_{2}$ is the class of all transpositions, $\rho=\chi_{ \pm}$is some character and $n=3,4,5$, then the dimension of $\mathfrak{B}(\mathcal{C}, \rho)$ is finite. See [FK, MS].

- If $\mathcal{C}=\mathcal{O}_{4}$ is the class of all 4-cycles in $\mathbb{S}_{4}$ and $\rho=\chi_{-1}$ is some character, then the dimension of $\mathfrak{B}(\mathcal{C}, \rho)$ is finite [AG2, Th. 6.12].

It is a hard open problem to determine if the dimension of $\mathfrak{B}\left(\mathcal{O}_{2}, \chi_{ \pm}\right)$is finite or not for $n \geq 6$. The technique in the present paper does not contribute to this question, since all braided subspaces of diagonal type of $M\left(\mathcal{O}_{2}, \chi_{ \pm}\right)$give rise to Nichols algebras of finite dimension (actually exterior algebras). We are chiefly concerned with other representations or other orbits, hoping that a better understanding of the special features of $M\left(\mathcal{O}_{2}, \chi_{ \pm}\right)$(by contrast with the other orbits) would permit us to isolate those conditions that are right for consideration of this hard problem.

The main results in this paper are summarized in the following statement.

Theorem 1. Let $\pi \in \mathbb{S}_{n}$. If either one of the following conditions holds:

(i) the order of $\pi$ is odd, or

(ii) all cycles in $\pi$ have odd order except for exactly two transpositions, then $\operatorname{dim} \mathfrak{B}\left(\mathcal{O}_{\pi}, \rho\right)=\infty$ for any $\rho \in \widehat{\mathbb{S}_{n}^{\pi}}$.

See Theorems 2.4 and 2.7. We also apply our main result to determine all irreducible Yetter-Drinfeld modules over $\mathbb{S}_{3}$ or $\mathbb{S}_{4}$ whose Nichols algebra is finitedimensional. See Theorem 2.8 .

\section{Preliminaries and Conventions}

Our references for pointed Hopf algebras are [M], AS3]. The set of isomorphism classes of irreducible representations of a finite group $G$ is denoted by $\widehat{G}$; thus, the group of characters of a finite abelian group $\Gamma$ is denoted by $\widehat{\Gamma}$. We shall often confuse a representative of a class in $\widehat{G}$ with the class itself. If $V$ is a $\Gamma$-module, then $V^{\chi}$ denotes the isotypic component of type $\chi \in \widehat{\Gamma}$. It is useful to write $g \triangleright h=g h g^{-1}$, $g, h \in G$.

1.1. Notation on the groups $\mathbb{S}_{n}$. Recall that the type of a permutation $\pi \in \mathbb{S}_{n}$ is a symbol $\left(1^{m_{1}}, 2^{m_{2}}, \ldots, n^{m_{n}}\right)$ meaning that in the decomposition of $\pi$ as a product of disjoint cycles, there are $m_{j}$ cycles of length $j, 1 \leq j \leq n$. We may omit $j^{m_{j}}$ when $m_{j}=0$. The conjugacy class $\mathcal{O}_{\pi}$ of $\pi$ coincides with the set of all permutations in

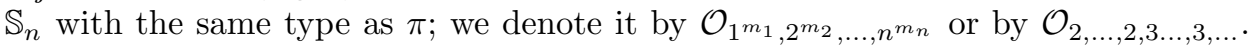
For instance, we denote by $\mathcal{O}_{j}$ the conjugacy class of $j$-cycles in $\mathbb{S}_{n}, 2 \leq j \leq n$. Also, $\mathcal{O}_{2,2}$ is the conjugacy class of (12)(34) and so on. If $\pi \in \mathbb{S}_{n}$ and $n<m$, then we also denote by $\pi$ the natural extension to $\mathbb{S}_{m}$ that fixes all $i>n$. If some emphasis is needed, we add a superscript $n$ to indicate that we are taking conjugacy classes in $\mathbb{S}_{n}$, e.g., $\mathcal{O}_{j}^{n}$ for the conjugacy class of $j$-cycles in $\mathbb{S}_{n}$. It is well known that the isotropy subgroup $\mathbb{S}_{n}^{\pi}$ is isomorphic to a product

$$
\mathbb{S}_{n}^{\pi} \simeq T_{1} \ldots T_{n},
$$

where $T_{i}=\Gamma_{i} \rtimes \mathbb{S}_{m_{i}}, 1 \leq i \leq n$. Here $\Gamma_{i} \simeq(\mathbb{Z} / i)^{m_{i}}$ is generated by the $i$-cycles in $\pi$ and $\mathbb{S}_{m_{i}}$ permutes these cycles. Hence any $\rho \in \widehat{\mathbb{S}_{n}^{\pi}}$ is of the form $\rho_{1} \otimes \cdots \otimes \rho_{n}$ where $\rho_{i} \in \widehat{T}_{i}$.

If $X$ is a subset of $\{1, \ldots, n\}$, then $\mathbb{S}_{X}$ denotes the subgroup of bijections in $\mathbb{S}_{n}$ that fix pointwise $\{1, \ldots, n\}-X$. We shall use the following notation for representations of subgroups of $\mathbb{S}_{n}$. Let $\omega_{j}$ be a fixed primitive $j$-th root of $1,2 \leq j \leq n$, 
and let $\tau_{j}=(123 \ldots j)$. Define

$$
\begin{aligned}
\varepsilon & =\text { trivial character; } \\
\text { sgn } & =\text { sign character of } \mathbb{S}_{X}, X \subset \mathbb{S}_{n} ; \\
\chi_{j} & =\text { character of }\left\langle\tau_{j}\right\rangle \simeq \mathbb{Z}_{j} \text { given by } \chi\left(\tau_{j}\right)=\omega_{j} .
\end{aligned}
$$

1.2. Yetter-Drinfeld modules over the group algebra of a finite group. A Yetter-Drinfeld module over a finite group $G$ is a left $G$-module and a left $\mathbb{C} G$ comodule $M$ such that

$$
\delta(g \cdot m)=g h g^{-1} \otimes g . m, \quad m \in M_{h}, g, h \in G .
$$

Here $M_{h}=\{m \in M: \delta(m)=h \otimes m\}$; clearly, $M=\bigoplus_{h \in G} M_{h}$. Yetter-Drinfeld modules over $G$ are completely reducible. Also, irreducible Yetter-Drinfeld modules over $G$ are parameterized by pairs $(\mathcal{C}, \rho)$ where $\mathcal{C}$ is a conjugacy class and $\rho$ is an irreducible representation of the isotropy subgroup $G^{s}$ of a fixed point $s \in \mathcal{C}$. As usual, $\operatorname{deg} \rho$ is the dimension of the vector space $V$ affording $\rho$. We denote the corresponding Yetter-Drinfeld module by $M(\mathcal{C}, \rho)$; see [DPR, W] and also AG1. Since $s \in Z\left(G^{s}\right)$, the Schur Lemma says that

$$
s \text { acts by a scalar } q_{s s} \text { on } V \text {. }
$$

Here is a precise description of the Yetter-Drinfeld module $M(\mathcal{C}, \rho)$. Let $t_{1}=s$, $\ldots, t_{M}$ be a numeration of $\mathcal{C}$ and let $g_{i} \in G$ such that $g_{i} \triangleright s=t_{i}$ for all $1 \leq i \leq M$. Then $M(\mathcal{C}, \rho)=\bigoplus_{1 \leq i \leq M} g_{i} \otimes V$. Let $g_{i} v:=g_{i} \otimes v \in M(\mathcal{C}, \rho), 1 \leq i \leq M, v \in V$. If $v \in V$ and $1 \leq i \leq M$, then the action of $g \in G$ and the coaction are given by

$$
\delta\left(g_{i} v\right)=t_{i} \otimes g_{i} v, \quad g \cdot\left(g_{i} v\right)=g_{j}(\gamma \cdot v),
$$

where $g g_{i}=g_{j} \gamma$, for some $1 \leq j \leq M$ and $\gamma \in G^{s}$. The explicit formula for the braiding is then given by

$$
c\left(g_{i} v \otimes g_{j} w\right)=t_{i} \cdot\left(g_{j} w\right) \otimes g_{i} v=g_{h}(\gamma \cdot v) \otimes g_{i} v
$$

for any $1 \leq i, j \leq M, v, w \in V$, where $t_{i} g_{j}=g_{h} \gamma$ for unique $h, 1 \leq h \leq M$ and $\gamma \in G^{s}$.

1.3. On Nichols algebras. Let $(V, c)$ be a braided vector space; that is, $V$ is a vector space and $c: V \otimes V \rightarrow V \otimes V$ is a linear isomorphism satisfying the braid equation. Then $\mathfrak{B}(V)$ denotes the Nichols algebra of $V$; see the precise definition in AS3. Let $G$ be a finite group, $\mathcal{C}$ a conjugacy class, $s \in \mathcal{C}$ and $\rho \in \widehat{G^{s}}$. The Nichols algebra of $M(\mathcal{C}, \rho)$ will be denoted simply by $\mathfrak{B}(\mathcal{C}, \rho)$. We collect some general facts on Nichols algebras for further reference.

Remark 1.1. Let $(V, c)$ be a braided vector space. If $W$ is a subspace of $V$ such that $c(W \otimes W)=W \otimes W$, then $\mathfrak{B}(W) \subset \mathfrak{B}(V)$. Thus $\operatorname{dim} \mathfrak{B}(W)=\infty \Longrightarrow$ $\operatorname{dim} \mathfrak{B}(V)=\infty$. In particular, if there exists $v \in V-0$ such that $c(v \otimes v)=v \otimes v$, then $\operatorname{dim} \mathfrak{B}(V)=\infty$. This is the case in $\mathfrak{B}(\mathcal{C}, \rho)$ when either the orbit $\mathcal{C}$ is trivial or the representation $\rho$ is trivial, or even if the scalar $q_{s s}$ defined in (1) is 1 .

A braided vector space $(V, c)$ is of diagonal type if there exists a basis $v_{1}, \ldots, v_{\theta}$ of $V$ and non-zero scalars $q_{i j}, 1 \leq i, j \leq \theta$, such that

$$
c\left(v_{i} \otimes v_{j}\right)=q_{i j} v_{j} \otimes v_{i}, \quad \text { for all } 1 \leq i, j \leq \theta .
$$


A braided vector space $(V, c)$ is of Cartan type if it is of diagonal type and there exists $a_{i j} \in \mathbb{Z},-$ ord $q_{i i}<a_{i j} \leq 0$ such that

$$
q_{i j} q_{j i}=q_{i i}^{a_{i j}}
$$

for all $1 \leq i \neq j \leq \theta$. Set $a_{i i}=2$ for all $1 \leq i \leq \theta$. Then $\left(a_{i j}\right)_{1 \leq i, j \leq \theta}$ is a generalized Cartan matrix. The following important result was proved in [H], Th. 4], showing that some hypotheses in [AS2, Th. 1] were unnecessary.

Theorem 1.2. Let $(V, c)$ be a braided vector space of Cartan type. Assume that $q_{i i} \neq 1$ is a root of 1 for all $1 \leq i \leq \theta$. Then $\operatorname{dim} \mathfrak{B}(V)<\infty$ if and only if the Cartan matrix is of finite type.

\section{On Nichols algebras over $\mathbb{S}_{n}$}

In this section we state some general results about Nichols algebras over $\mathbb{S}_{n}$. Our main idea is to find suitable braided subspaces of a Yetter-Drinfeld module over $\mathbb{C S}_{n}$ that are diagonal of Cartan type, so that Theorem 1.2 applies. In what follows, $G$ is a finite group, $s \in G, \mathcal{C}$ is the conjugacy class of $s, \rho \in \widehat{G^{s}}, \rho: G^{s} \rightarrow G L(V)$. Recall the scalar $q_{s s}$ defined in (11).

Proposition 2.1 (G1, Lemma 3.1]). Assume that $\operatorname{dim} \mathfrak{B}(\mathcal{C}, \rho)<\infty$. Then

- $\operatorname{deg} \rho>2$ implies $q_{s s}=-1$,

- $\operatorname{deg} \rho=2$ implies $q_{s s}=-1, \omega_{3}$ or $\omega_{3}^{2}$.

2.1. Nichols algebras corresponding to permutations of odd order. We begin with a general method of finding braided subspaces of rank 2. Assume that

$$
\text { there exists } \sigma \in G \text { such that } \sigma s \sigma^{-1}=s^{-1} \neq s .
$$

Hence $s^{-1} \in \mathcal{C}$, i.e., $\mathcal{C}$ is real. Under this hypothesis, we prove the following result that, unlike Proposition 2.1, does not assume any restriction on $\operatorname{deg} \rho$.

Lemma 2.2. If $\operatorname{dim} \mathfrak{B}(\mathcal{C}, \rho)<\infty$, then $q_{s s}=-1$ and $s$ has even order.

Proof. Let $N=\operatorname{ord} q_{s s}$; clearly $N>1$. Let $t_{1}=s, t_{2}=s^{-1}, g_{1}=e, g_{2}=\sigma$. By (3), $\sigma s^{-1} \sigma^{-1}=s$; thus $\sigma s^{-1}=s \sigma$ and $\sigma s=s^{-1} \sigma$. Together with (11) and (21), this shows that for any $v, w \in V$,

$$
\begin{aligned}
& c\left(g_{1} v \otimes g_{1} w\right)=g_{1}(s \cdot w) \otimes g_{1} v=q_{s s} g_{1} w \otimes g_{1} v, \\
& c\left(g_{1} v \otimes g_{2} w\right)=g_{2}\left(s^{-1} \cdot w\right) \otimes g_{1} v=q_{s s}^{-1} g_{2} w \otimes g_{1} v, \\
& c\left(g_{2} v \otimes g_{1} w\right)=g_{1}\left(s^{-1} \cdot w\right) \otimes g_{2} v=q_{s s}^{-1} g_{1} w \otimes g_{2} v, \\
& c\left(g_{2} v \otimes g_{2} w\right)=g_{2}(s \cdot w) \otimes g_{2} v=q_{s s} g_{2} w \otimes g_{2} v .
\end{aligned}
$$

Let $v \in V, v \neq 0$. Then the subspace of $M(\mathcal{C}, \rho)$ spanned by $v_{1}:=g_{1} v, v_{2}:=g_{2} v$ is a braided subspace of Cartan type: $c\left(v_{i} \otimes v_{j}\right)=q_{i j} v_{j} \otimes v_{i}$, for $1 \leq i, j \leq 2$, where $\left(\begin{array}{ll}q_{11} & q_{12} \\ q_{21} & q_{22}\end{array}\right)=\left(\begin{array}{cc}q_{s s} & q_{s s}^{-1} \\ q_{s s}^{-1} & q_{s s}\end{array}\right)$, with Cartan matrix $\left(\begin{array}{cc}2 & a_{12} \\ a_{21} & 2\end{array}\right), a_{12}=a_{21} \equiv$ $-2 \bmod N$. Now $a_{12}=a_{21}=0$ or -1 , by hypothesis and Theorem 1.2. Thus, necessarily $a_{12}=a_{21}=0$ and $N=2$.

Lemma 2.3. Let $\pi \in \mathbb{S}_{n}$, ord $\pi>2, \rho \in \widehat{\mathbb{S}_{n}^{\pi}}$. If $\operatorname{dim} \mathfrak{B}\left(\mathcal{O}_{\pi}, \rho\right)<\infty$, then $q_{\pi, \pi}=-1$. 
Proof. It is well known that (3) holds for any $\pi \in \mathbb{S}_{n}$. Namely, assume that $\pi=t_{j}$ for some $j$ and take

$$
g_{2}= \begin{cases}(1 j-1)(2 j-2) \cdots(k-1 k+1), & \text { if } j=2 k \text { is even, } \\ (1 j-1)(2 j-2) \cdots(k k+1), & \text { if } j=2 k+1 \text { is odd. }\end{cases}
$$

It is easy to see that $g_{2} t_{j} g_{2}^{-1}=t_{j}^{-1}$. The general case follows using that any $\pi$ is a product of disjoint cycles. With Lemma 2.2, this completes the proof.

Theorem 2.4. If $\pi \in \mathbb{S}_{n}$ has odd order, then $\operatorname{dim} \mathfrak{B}\left(\mathcal{O}_{\pi}, \rho\right)=\infty$ for any $\rho \in$ $\widehat{\mathbb{S}_{n}^{\pi}}$.

If $n$ is even, the Nichols algebras $\mathfrak{B}\left(\mathcal{O}_{n}^{n}, \rho\right)$ cannot be treated by similar arguments as above. For, the isotropy subgroup $\mathbb{S}_{n}^{\tau_{n}}$ is cyclic of order $n$ and we can assume that $\rho\left(\tau_{n}\right)=-1$. Assume that $\tau \in \mathcal{O}_{n}^{n} \cap \mathbb{S}_{n}^{\tau_{n}}$. Then $\tau=\tau_{n}^{j}$ with $(n, j)=1$; hence $\rho(\tau)=-1$. But there are Nichols algebras like these that are finite-dimensional. For instance, the Nichols algebra $\mathfrak{B}\left(\mathcal{O}_{4}, \chi_{4}^{2}\right)$ was computed in [AG2, Th. 6.12] and has dimension 576.

2.2. A reduction argument. We now discuss a general reduction argument. Let $n, p \in \mathbb{N}$ and let $m=n+p$. Let $\#: \mathbb{S}_{n} \times \mathbb{S}_{p} \rightarrow \mathbb{S}_{m}$ be the group homomorphism given by

$$
\pi \# \tau(i)= \begin{cases}\pi(i), & 1 \leq i \leq n \\ \tau(i-n)+n, & n+1 \leq i \leq m,\end{cases}
$$

$\pi \in \mathbb{S}_{n}, \tau \in \mathbb{S}_{p}$. If also $g \in \mathbb{S}_{n}, h \in \mathbb{S}_{p}$, then $(g \# h) \triangleright(\pi \# \tau)=(g \triangleright \pi) \#(h \triangleright \tau)$. Thus $\mathcal{O}_{\pi \# \tau} \supset \mathcal{O}_{\pi} \# \mathcal{O}_{\tau}$. Let us say that $\pi$ and $\tau$ are orthogonal, denoted $\pi \perp \tau$, if there is no $j$ such that both $\pi$ and $\tau$ contain a $j$-cycle. In other words, if $\pi$ has type $\left(1^{a_{1}}, 2^{a_{2}}, \ldots, n^{a_{n}}\right)$ and $\tau$ has type $\left(1^{b_{1}}, 2^{b_{2}}, \ldots, p^{b_{p}}\right)$, then either $a_{j}=0$ or $b_{j}=0$ for any $j$. If $\pi \perp \tau$, then $\mathbb{S}_{m}^{\pi \# \tau}=\mathbb{S}_{n}^{\pi} \# \mathbb{S}_{p}^{\tau}$, say by a counting argument. Hence any $\mu \in \widehat{\mathbb{S}_{m}^{\pi \# \tau}}$ is of the form $\mu=\rho \otimes \lambda$, for unique $\rho \in \widehat{\mathbb{S}_{n}^{\pi}}, \lambda \in \widehat{\mathbb{S}_{p}^{\tau}}$. We say that $V, W$ are the vector spaces affording $\rho, \lambda$. Let $q_{\tau \tau}$, resp. $q_{\pi \pi}$, be the scalar as in (1) for the representation $\rho$, resp. $\lambda$.

Lemma 2.5. Let $\pi \in \mathbb{S}_{n}, \tau \in \mathbb{S}_{p}$.

(1) Assume that $\pi \perp \tau$ and $\operatorname{ord}(\pi \# \tau)>2$. Let $\mu \in \widehat{\mathbb{S}_{m}^{\pi \# \tau}}$ be of the form $\mu=\rho \otimes \lambda$, for $\rho \in \widehat{\mathbb{S}_{n}^{\pi}}, \lambda \in \widehat{\mathbb{S}_{p}^{\tau}}$. If $\operatorname{dim} \mathfrak{B}\left(\mathcal{O}_{\pi \# \tau}, \mu\right)<\infty$, then $q_{\pi \pi} q_{\tau \tau}=-1$.

(2) Assume that the orders of $\pi$ and $\tau$ are relatively prime, that ord $q_{\tau \tau}$ is odd and that $\operatorname{ord}(\pi \# \tau)>2$. If $\operatorname{dim} \mathfrak{B}\left(\mathcal{O}_{\pi \# \tau}, \mu\right)<\infty$, then $q_{\tau \tau}=1$.

Clearly, if the orders of $\pi$ and $\tau$ are relatively prime, then $\pi \perp \tau$. On the other hand, if $\operatorname{ord}(\pi \# \tau)=2$ and the orders of $\pi$ and $\tau$ are relatively prime, then we can assume $\tau=e$, and $q_{\tau \tau}=1$ anyway.

Proof. Since $q_{\pi \# \tau, \pi \# \tau}=q_{\pi, \pi} q_{\tau \tau}$, (1) follows from Lemma 2.3. Then (2) follows from (1).

Our aim is to obtain information on $\mathfrak{B}\left(\mathcal{O}_{\pi \# \tau}, \mu\right)$ from $\mathfrak{B}\left(\mathcal{O}_{\pi}, \rho\right)$ and $\mathfrak{B}\left(\mathcal{O}_{\tau}, \lambda\right)$. For this, we fix $g_{1}=e, \ldots, g_{P} \in \mathbb{S}_{n}, h_{1}=e, \ldots, h_{T} \in \mathbb{S}_{p}$, such that

$$
\begin{aligned}
& g_{1} \triangleright \pi=\pi, \ldots, g_{P} \triangleright \pi \text { is a numeration of } \mathcal{O}_{\pi}, \\
& h_{1} \triangleright \tau=\tau, \ldots, h_{T} \triangleright \tau \text { is a numeration of } \mathcal{O}_{\tau} .
\end{aligned}
$$


Then we can extend $\left(g_{i} \# h_{j}\right) \triangleright(\pi \# \tau), 1 \leq i \leq P, 1 \leq j \leq T$ to a numeration of $\mathcal{O}_{\pi \# \tau}$. Let $v, u \in V, w, z \in W, 1 \leq i, k \leq P, 1 \leq j, l \leq T$. Then the braiding in $M\left(\mathcal{O}_{\pi \# \tau}, \rho \otimes \lambda\right)$ has the form

$$
\begin{aligned}
& c\left(\left(g_{i} \# h_{j}\right)(v \otimes w) \otimes\left(g_{k} \# h_{l}\right)(u \otimes z)\right) \\
& \quad=\left(\left(g_{r} \# h_{p}\right)(\gamma \cdot u \otimes \beta \cdot z) \otimes\left(g_{i} \# h_{j}\right)(v \otimes w)\right),
\end{aligned}
$$

where $\left(g_{i} \triangleright \pi\right) g_{k}=g_{r} \gamma,\left(h_{j} \triangleright \tau\right) h_{l}=h_{p} \beta$ for unique $1 \leq r \leq P, 1 \leq p \leq T, \gamma \in \mathbb{S}_{n}^{\pi}$ and $\beta \in \mathbb{S}_{p}^{\tau}$. Assume in (4) that $j=l=1$; then $p=1, \beta=\tau$ and (4) takes the form

$$
\begin{aligned}
& c\left(\left(g_{i} \# h_{1}\right)(v \otimes w) \otimes\left(g_{k} \# h_{1}\right)(u \otimes z)\right) \\
& \quad=q_{\tau \tau}\left(\left(g_{r} \# h_{1}\right)(\gamma \cdot u \otimes z) \otimes\left(g_{i} \# h_{1}\right)(v \otimes w)\right) .
\end{aligned}
$$

Our aim is to spell out some consequences of formula (5).

Proposition 2.6. Let $\pi \in \mathbb{S}_{n}, \tau \in \mathbb{S}_{p}$. Assume that the orders of $\pi$ and $\tau$ are relatively prime and that ord $q_{\tau \tau}$ is odd. Let $\mu \in \widehat{\mathbb{S}_{m}^{\pi \# \tau}}$ be of the form $\mu=\rho \otimes \lambda$, for $\rho \in \widehat{\mathbb{S}_{n}^{\pi}}, \lambda \in \widehat{\mathbb{S}_{p}^{\tau}}$

(1) If $\operatorname{dim} \mathfrak{B}\left(\mathcal{O}_{\pi \# \tau}, \mu\right)<\infty$, then $\operatorname{dim} \mathfrak{B}\left(\mathcal{O}_{\pi}, \rho\right)<\infty$.

(2) If $\operatorname{dim} \mathfrak{B}\left(\mathcal{O}_{\pi}, \rho\right)=\infty$ for any $\rho \in \widehat{\mathbb{S}_{n}^{\pi}}$, then $\operatorname{dim} \mathfrak{B}\left(\mathcal{O}_{\pi \# \tau}, \mu\right)=\infty$ for any $\mu \in \widehat{\mathbb{S}_{m}^{\pi \# \tau}}$.

In particular, let $\pi \in \mathbb{S}_{n}$ with no fixed points. If $\operatorname{dim} \mathfrak{B}\left(\mathcal{O}_{\pi}^{n}, \rho\right)=\infty$ for any $\rho \in \widehat{\mathbb{S}_{n}^{\pi}}$, then $\operatorname{dim} \mathfrak{B}\left(\mathcal{O}_{\pi}^{m}, \rho^{\prime}\right)=\infty$ for any $\rho^{\prime} \in \widehat{\mathbb{S}_{m}^{\pi}}, m>n$.

Proof. Assume that $\operatorname{dim} \mathfrak{B}\left(\mathcal{O}_{\pi \# \tau}, \mu\right)<\infty$. By Lemma 2.5, $q_{\tau \tau}=1$. Let $0 \neq$ $w=z \in W$. The linear map $\psi: M\left(\mathcal{O}_{\pi}, \rho\right) \rightarrow M\left(\mathcal{O}_{\pi \# \tau}, \mu\right)$ given by $\psi\left(g_{i} v\right)=$ $\left(g_{i} \# h_{1}\right)(v \otimes w)$ is a morphism of braided vector spaces because of (5). Now apply Remark 1.1 .

\subsection{Nichols algebras of orbits with exactly two transpositions.}

Theorem 2.7. Let $\pi \in \mathbb{S}_{n}$. If $\pi$ has type $\left(1^{a}, 2^{2}, h_{1}^{m_{1}}, \ldots, h_{r}^{m_{r}}\right)$ where $h_{1}, \ldots, h_{r}$ are odd, then $\operatorname{dim} \mathfrak{B}\left(\mathcal{O}_{\pi}^{n}, \rho\right)=\infty$ for any $\rho \in \widehat{\mathbb{S}_{n}^{\pi}}$.

For instance, if $n \geq 4$, then $\operatorname{dim} \mathfrak{B}\left(\mathcal{O}_{2,2}^{n}, \rho\right)=\infty$ for any $\rho$.

Proof. By Proposition 2.6 we can assume that $n=4$ and $\pi$ is of type $(2,2)$. Let us consider the irreducible Yetter-Drinfeld modules corresponding to $\mathcal{O}_{2,2}=$ $\{a=(13)(24), b=(12)(34), d=(14)(23)\}$. The isotropy subgroup of $a$ is $\mathbb{S}_{4}^{a}=$ $\langle(1234),(13)\rangle \simeq \mathcal{D}_{4}$. Let $A=(1234), B=(13) ; \mathcal{O}_{2,2} \subseteq \mathbb{S}_{4}^{a}$ and $a=A^{2}, b=B A$, $d=B A^{3}$. Hence the irreducible representations of $\mathbb{S}_{4}^{a}$ are (1) the characters given by $A \mapsto \varepsilon_{1}, B \mapsto \varepsilon_{2}$ where $\varepsilon_{j} \in\{ \pm 1\}, 1 \leq j \leq 2$, and (2) the 2-dimensional representation $\rho: \mathbb{D}_{4} \rightarrow G L(2, \mathbb{C})$ given by

$$
\rho(A)=\left(\begin{array}{cc}
0 & -1 \\
1 & 0
\end{array}\right), \quad \rho(B)=\left(\begin{array}{cc}
-1 & 0 \\
0 & 1
\end{array}\right) .
$$

Let $\mu$ be a one-dimensional representation of $\mathbb{S}_{4}^{a}$. Then $M\left(\mathcal{O}_{2,2}, \mu\right)$ has a basis $v_{a}, v_{b}, v_{d}$ with $\delta\left(v_{a}\right)=a \otimes v_{a}$, etc., and $c\left(v_{a} \otimes v_{a}\right)=\mu(a) v_{a} \otimes v_{a}=v_{a} \otimes v_{a}$. Hence $\operatorname{dim} \mathfrak{B}\left(\mathcal{O}_{2,2}, \mu\right)=\infty$. 
Let $\sigma_{0}=1, \sigma_{1}=(12), \sigma_{2}=(23)$. Then

$$
\sigma_{1} \triangleright a=d, \quad \sigma_{2} \triangleright a=b, \quad \sigma_{1} \triangleright b=b, \quad \sigma_{2} \triangleright d=d .
$$

Let us consider the Yetter-Drinfeld module $M\left(\mathcal{O}_{2,2}, \rho\right)$. We have

$$
\rho(a)=\left(\begin{array}{cc}
-1 & 0 \\
0 & -1
\end{array}\right), \quad \rho(b)=\left(\begin{array}{ll}
0 & 1 \\
1 & 0
\end{array}\right), \quad \rho(d)=\left(\begin{array}{cc}
0 & -1 \\
-1 & 0
\end{array}\right) .
$$

Let $\sigma_{j} v:=\sigma_{j} \otimes v, v \in V, 0 \leq j \leq 2$. The coaction is given by $\delta\left(\sigma_{j} v\right)=\sigma_{j} \triangleright a \otimes \sigma_{j} v$; we need the action of the elements $a, b, d$, which is

$$
\begin{aligned}
& a \cdot \sigma_{0} v=\sigma_{0} \rho(a)(v), \quad a \cdot \sigma_{1} v=\sigma_{1} \rho(d)(v), \quad a \cdot \sigma_{2} v=\sigma_{2} \rho(b)(v), \\
& b \cdot \sigma_{0} v=\sigma_{0} \rho(b)(v), \quad b \cdot \sigma_{1} v=\sigma_{1} \rho(b)(v), \quad b \cdot \sigma_{2} v=\sigma_{2} \rho(a)(v), \\
& d \cdot \sigma_{0} v=\sigma_{0} \rho(d)(v), \quad d \cdot \sigma_{1} v=\sigma_{1} \rho(a)(v), \quad d \cdot \sigma_{2} v=\sigma_{2} \rho(d)(v) \text {. }
\end{aligned}
$$

Hence the braiding is given, for all $0 \leq j \leq 2$ and $v, w \in V$, by

$$
c\left(\sigma_{j} v \otimes \sigma_{j} w\right)=\left(\sigma_{j} \triangleright a\right) \cdot \sigma_{j} w \otimes \sigma_{j} v=\sigma_{j} \rho(a)(w) \otimes \sigma_{j} v=-\sigma_{j} w \otimes \sigma_{j} v
$$

and

$$
\begin{array}{llrl}
c\left(\sigma_{0} v \otimes \sigma_{1} w\right) & =\sigma_{1} \rho(d)(w) \otimes \sigma_{0} v, & & c\left(\sigma_{0} v \otimes \sigma_{2} w\right)=\sigma_{2} \rho(b)(w) \otimes \sigma_{0} v, \\
c\left(\sigma_{1} v \otimes \sigma_{0} w\right) & =\sigma_{0} \rho(d)(w) \otimes \sigma_{1} v, & & c\left(\sigma_{1} v \otimes \sigma_{2} w\right)=\sigma_{2} \rho(d)(w) \otimes \sigma_{1} v, \\
c\left(\sigma_{2} v \otimes \sigma_{0} w\right) & =\sigma_{0} \rho(b)(w) \otimes \sigma_{2} v, & & c\left(\sigma_{2} v \otimes \sigma_{1} w\right)=\sigma_{1} \rho(b)(w) \otimes \sigma_{2} v .
\end{array}
$$

Let $v_{1}=\left(\begin{array}{l}1 \\ 1\end{array}\right), v_{2}=\left(\begin{array}{c}1 \\ -1\end{array}\right)$. Then $\rho(b)\left(v_{1}\right)=v_{1}, \rho(b)\left(v_{2}\right)=-v_{2}, \rho(d)\left(v_{1}\right)=-v_{1}$, $\rho(d)\left(v_{2}\right)=v_{2}$. Hence the braiding is diagonal of Cartan type in the basis

$$
w_{1}=\sigma_{0} v_{1}, w_{2}=\sigma_{0} v_{2}, w_{3}=\sigma_{1} v_{1}, w_{4}=\sigma_{1} v_{2}, w_{5}=\sigma_{2} v_{1}, w_{6}=\sigma_{2} v_{2} .
$$

The corresponding Dynkin diagram is not connected; its connected components are $\{1,4,6\}$ and $\{2,3,5\}$, each of them supporting the affine Dynkin diagram $A_{2}^{(1)}$. Then $\operatorname{dim} \mathfrak{B}\left(\mathcal{O}_{2,2}, \rho\right)=\infty$ by Theorem 1.2 .

TABLE 1. Nichols algebras of irreducible Yetter-Drinfeld modules over $\mathbb{S}_{3}$

\begin{tabular}{|l|l|l|l|l|}
\hline Orbit & $\begin{array}{l}\text { Isotropy } \\
\text { group }\end{array}$ & Representation & $\operatorname{dim} \mathfrak{B}(V)$ & Reference \\
\hline$e$ & $\mathbb{S}_{3}$ & any & $\infty$ & Remark [.1 \\
\hline $\mathcal{O}_{3}$ & $\mathbb{Z}_{3}$ & any & $\infty$ & Theorem [2.4 \\
\hline $\mathcal{O}_{2}$ & $\mathbb{Z}_{2}$ & $\varepsilon$ & $\infty$ & Remark [.1 \\
\hline $\mathcal{O}_{2}$ & $\mathbb{Z}_{2}$ & sgn & 12 & {$[$ MS } \\
\hline
\end{tabular}


TABLE 2. Nichols algebras of irreducible Yetter-Drinfeld modules over $\mathbb{S}_{4}$

\begin{tabular}{|l|l|l|l|l|}
\hline Orbit & $\begin{array}{l}\text { Isotropy } \\
\text { group }\end{array}$ & Representation & $\operatorname{dim} \mathfrak{B}(V)$ & Reference \\
\hline$e$ & $\mathbb{S}_{4}$ & any & $\infty$ & Remark [1.1] \\
\hline $\mathcal{O}_{2,2}$ & $\mathcal{D}_{4}$ & any & $\infty$ & Theorem [2.7 \\
\hline $\mathcal{O}_{4}$ & $\mathbb{Z}_{4}$ & $\varepsilon$ & $\infty$ & Remark [1.1] \\
\hline $\mathcal{O}_{4}$ & $\mathbb{Z}_{4}$ & $\chi_{4}$ or $\chi_{4}^{3}$ & $\infty$ & Lemma [2.3 \\
\hline $\mathcal{O}_{4}$ & $\mathbb{Z}_{4}$ & $\chi_{4}^{2}$ & 576 & {$[$ AG2, 6.12] } \\
\hline $\mathcal{O}_{3}$ & $\mathbb{Z}_{3}$ & any & $\infty$ & Theorem [2.4 \\
\hline $\mathcal{O}_{2}$ & $\mathbb{Z}_{2} \oplus \mathbb{Z}_{2}$ & $\varepsilon$ or $\varepsilon \oplus \operatorname{sgn}$ & $\infty$ & Remark [1.1 \\
\hline $\mathcal{O}_{2}$ & $\mathbb{Z}_{2} \oplus \mathbb{Z}_{2}$ & $\operatorname{sgn} \oplus \varepsilon$ & 576 & {$[$ FK] } \\
\hline $\mathcal{O}_{2}$ & $\mathbb{Z}_{2} \oplus \mathbb{Z}_{2}$ & $\operatorname{sgn} \oplus \operatorname{sgn}$ & 576 & {$[$ MS $]$} \\
\hline
\end{tabular}

2.4. Nichols algebras over $\mathbb{S}_{3}$ and $\mathbb{S}_{4}$. We apply the main result of this paper to classify finite-dimensional Nichols algebras over $\mathbb{S}_{3}$ or $\mathbb{S}_{4}$ with an irreducible module of primitive elements.

Theorem 2.8. Let $M(\mathcal{C}, \lambda)$ be an irreducible Yetter-Drinfeld module over $\mathbb{S}_{n}$ such that $\mathfrak{B}(\mathcal{C}, \lambda)$ is finite-dimensional.

(i). If $\mathbb{S}_{n}=\mathbb{S}_{3}$, then $M(\mathcal{C}, \lambda) \simeq M\left(\mathcal{O}_{2}, \mathrm{sgn}\right)$.

(ii). If $\mathbb{S}_{n}=\mathbb{S}_{4}$, then $M(\mathcal{C}, \lambda)$ is isomorphic either to $\mathfrak{B}\left(\mathcal{O}_{4}, \chi_{4}^{2}\right)$ or to $\mathfrak{B}\left(\mathcal{O}_{2}\right.$, $\operatorname{sgn} \oplus \varepsilon)$ or to $\mathfrak{B}\left(\mathcal{O}_{2}, \operatorname{sgn} \oplus \operatorname{sgn}\right)$.

Proof. See Tables 1 and 2 .

\section{ACKNOWLEDGMENTS}

The first author thanks István Heckenberger and Matías Graña for interesting e-mail exchanges.

\section{REFERENCES}

[AG1] N. Andruskiewitsch and M. Graña, Braided Hopf algebras over non-abelian groups, Bol. Acad. Ciencias (Córdoba) 63 (1999), 45-78. Also in math.QA/9802074. MR:1714540 (2001b:16039)

[AG2] , From racks to pointed Hopf algebras, Adv. Math. 178, 177-243 (2003). MR:1994219 (2004i:16046)

[AS1] N. Andruskiewitsch and H.-J. Schneider, Lifting of Quantum Linear Spaces and Pointed Hopf Algebras of order $p^{3}$, J. Algebra 209 (1998), 658-691. MR.1659895 (99k:16075)

[AS2] - Finite quantum groups and Cartan matrices, Adv. Math. 154 (2000), 1-45. MR 1780094 (2001g:16070) 
[AS3] - Pointed Hopf Algebras, in "New directions in Hopf algebras", 1-68, Math. Sci. Res. Inst. Publ. 43, Cambridge Univ. Press, Cambridge, 2002. MR.1913436 (2003e:16043)

[AS4] - On the classification of finite-dimensional pointed Hopf algebras, preprint math.QA/0502157, Ann. Math., to appear.

[DPR] R. Dijkgraaf, V. Pasquier and P. Roche, Quasi Hopf algebras, group cohomology and orbifold models, Nuclear Phys. B Proc. Suppl. 18B (1991), pp. 60-72. MR1128130 (92m:81238)

[FK] S. Fomin and K. N. Kirillov, Quadratic algebras, Dunkl elements, and Schubert calculus, Progr. Math. 172, Birkhäuser, (1999), pp. 146-182. MR1667680(2001a:05152)

[G1] M. Graña, On Nichols algebras of low dimension, Contemp. Math. 267 (2000), pp. 111134. MR1800709 (2001j:16059)

$[\mathrm{H}] \quad$ I. Heckenberger, The Weyl groupoid of a Nichols algebra of diagonal type, math.QA/0411477, Inventiones Math. 164, 175-188 (2006). MR2207786

[MS] A. Milinski and H.-J. Schneider, Pointed Indecomposable Hopf Algebras over Coxeter Groups, Contemp. Math. 267 (2000), 215-236. MR.1800714(2001k:16074)

[M] S. Montgomery, Hopf Algebras and Their Actions on Rings, CBMS Conf. Series in Math., vol. 82, Amer. Math. Soc., Providence, RI, 1993. MR.1243637 (94i:16019)

[W] S. Witherspoon, The representation ring of the quantum double of a finite group, J. Algebra 179 (1996), 305-329. MR1367852(96m:20015)

Famaf, Universidad Nacional de Córdoba. Ciem - COniCet, (5000) Ciudad Universitaria, Córdoba, Argentina

E-mail address: andrus@mate.uncor.edu

Department of Mathematics, Hunan University, Changsha 410082, People's Republic OF CHINA

E-mail address: z9491@yahoo.com.cn 